\title{
Modellbasierte Umsetzung des Anforderungsmanagements zur Unterstützung der Entwicklung eines Experimentalsatelliten
}

\author{
Emir Gadzo ${ }^{1 *}$, Jan Mehlstäubl ${ }^{1}$, Martin Denk ${ }^{1}$, Kristin Paetzold $^{1}$ \\ ${ }^{1}$ Universität der Bundeswehr, Fakultät für Luft- und Raumfahrt, Institut für Technische Produktentwicklung \\ * Korrespondierender Autor: \\ Emir Gadzo \\ Universität der Bundeswehr \\ Fakultät für Luft- und Raumfahrt \\ Institut für Technische Produktentwicklung, LRT-3 \\ Werner-Heisenberg-Weg 39 \\ 85579 Neubiberg \\ Telefon: +49 89/6004-3263 \\ Mail:emir.gadzo@unibw.de
}

\begin{abstract}
The needs and expectations of customers for a system are reflected in the requirements. The management of requirements therefore plays an important role in the product development process. In order to address the increasing complexity in product development and the resulting impact on requirements management, a model-based approach for the implementation of requirements management is needed in a satellite research project. In this paper, previous approaches for the implementation of a model-based requirements management are systematically examined for their possibilities and limitations. Subsequently, a targetoriented concept for the model-based implemen-tation of requirements management for a satellite research project is subsequently derived.
\end{abstract}

\section{Keywords}

Model based systems engineering (MBSE), requirements management, satellite research project, model based approach, systems engineering 


\section{Einleitung}

Die Bedürfnisse und Erwartungen eines Kunden an ein System werden durch die Anforderungen wiedergegeben [1]. Das Management der Anforderungen nimmt daher eine wichtige Rolle im Produktentstehungsprozess ein. Die Tatsache, dass die Produktentwicklung mit einer zunehmenden Komplexität der zu entwickelnden Systeme konfrontiert wird [2], hat Auswirkungen auf das Anforderungsmanagement. Durch diese Komplexitätssteigerung hat sich auch der Umfang des Aufgabenfeldes des Anforderungsmanagements geändert [3]. Um nun die Bedürfnisse und Erwartungen eines Kunden an ein System zufrieden zu stellen, ist es notwendig, neben der Beherrschung der zunehmenden Komplexität auch einen Verständnismangel bezüglich der Anforderungen zu minimieren und eine ineffiziente Kommunikation im Entwicklungsprozess zu verhindern [4]. Es ist möglich, mithilfe eines modellbasierten Ansatzes alle drei Aspekte positiv zu beeinflussen [4]. Erkennen von Komplexität und Förderung von Verständnis, aber auch eine Verbesserung der Kommunikation lassen sich durch die Modellierung erreichen. Diese Punkte stellen auch die Kernkompetenzen eines modellbasierten Ansatzes für das Anforderungsmanagement dar. Des Weiteren bietet ein modellbasierter Ansatz im Vergleich zu einem dokumentenzentrierten Ansatz Vorteile wie eine reduzierte Entwicklungszeit, eine verbesserte Analysefähigkeit und ein erhöhtes Potenzial zur Wiederverwendung [5]. Ferner gilt nach [6], dass eine erfolgreiche Entwicklung eine formale Sprache und eine geeignete Methode benötigt. Bei der Entwicklung eines Experimentalsatelliten spielen diese Aspekte ebenfalls eine wichtige Rolle, da die Bedürfnisse und Anforderungen von vielen verschiedenen technischen Disziplinen und Interessensgruppen zu berücksichtigen sind. Grundsätzlich handelt es sich bei Satelliten um komplexe Systeme, gebaut und entwickelt in einem interdisziplinären Umfeld von verschiedenen Beitragsleistern. Sie bestehen aus einer hohen Anzahl an interagierenden Komponenten und Objekten, wie z.B. Software, Hardware, Menschen, Einrichtungen und Dienstleistungen [6]. Es leitet sich daraus die Problemstellung ab, während des gesamten Entwicklungsprozesses den Bedürfnissen und Anforderungen der unterschiedlichen Interessensgruppen mit einem ganzheitlichen Systemverständnis gerecht zu werden und dabei Abhängigkeiten und Schnittstellen zwischen den einzelnen Teillösungen des Experimentalsatelliten zu identifizieren und den Daten- und Informationsaustausch zu unterstützen. Diese Problemstellung führt zu einem zusätzlichen Auftreten von dynamischen Anforderungen, was mit einem klassischen Lastenheft-basierten Anforderungsmanagement eine weitere enorme Herausforderung darstellt. Das modellbasierte Anforderungsmanagement hingegen bietet einerseits die Möglichkeit dynamische Anforderungen über den gesamten Produktentstehungsprozess in einem Systemmodell zu verwalten und anderseits auch eine eindeutige Nachverfolgung und Kommunikation zu gewährleisten [7]. Daraus resultierend wird für die Umsetzung des Anforderungsmanagements und die Identifikation der damit einhergehenden Daten- und Informationsflüsse im Rahmen eines Satellitenforschungsprojektes ein modellbasierter Ansatz herangezogen. In diesem Paper wird die Forschungsfrage erörtert, welche Möglichkeiten und Einschränkungen bisherige Ansätze aus der Literatur zur Umsetzung eines modellbasierten Anforderungsmanagements in einem Satellitenprojekt bieten. Ziel ist es, auf Grundlage von elaborierten Kriterien festzustellen, was einen zielführenden Ansatz unter Berücksichtigung der Grundsätze des Systems-Engineering für das Satellitenforschungsprojekt darstellt und im Rahmen einer investigativen Literaturrecherche die Grundlagen für einen der Definition entsprechenden zielführenden Ansatz zu eruieren.

In Kapitel 2 wird zunächst der inhaltliche Hintergrund erläutert. Es werden die beiden Disziplinen MBSE und Anforderungsmanagement umrissen und die Motivation, Zielsetzung und der technische Inhalt eines Experimentalsatellitenprojektes beschrieben. Im dritten Kapitel wird das Forschungsvorgehen zur Auflösung beschrieben. Kapitel 4 beinhaltet die Definition der Bewertungskriterien, das Ergebnis der Bewertung und die Ableitung eines Ansatzes. Abschließend wird im fünten Kapitel eine Zusammenfassung und ein Ausblick abgegeben. 


\section{Hintergrund}

\subsection{Modellbasiertes Systems Engineering (MBSE)}

Der Begriff modellbasiertes Systems-Engineering setzt sich aus den beiden Fachtermini Modell und Systems-Engineering zusammen. Für den Begriff Systems-Engineering gibt es verschiedene Beschreibungsansätze, wobei es nach [8] bestimmte Eigenschaften gibt, die sich aus den verschiedenen Definitionen ergeben und den Begriff charakterisieren: Interdisziplinär, iterativ, ganzheitlich, soziotechnisch und vor allem das zugrundeliegende Systemdenken. In [8] wird das Systems-Engineering als ein interdisziplinärer Ansatz betrachtet, der die Umsetzung erfolgreicher Systeme ermöglicht und sich dabei unter anderem auf die Definition von Kundenbedürfnissen und der dabei benötigten Funktionalität fokussiert. Die gesamthafte Problemstellung wird dabei stets berücksichtigt. Der Fachterminus Modell lässt sich nach [9] als eine Abstraktion eines Systems verstehen. Das Modell fokussiert dabei die für eine definierte Betrachtung relevanten Aspekte eines Systems und vernachlässigt andere Aspekte im Gegenzug. Sie trennen das für eine bestimmte Aufgabe Wesentliche vom Unwesentlichen und werden unter anderem zur Erfassung und Analyse wichtiger Parameter eines Systems, sowie zur Schaffung eines besseren Problemverständnisses und der Dokumentation relevanter Systemmerkmale eingesetzt [10]. Der Einsatz eines Modells und die damit verbundene Modellierung bringen nach [9] folgende Vorteile und Möglichkeiten mit sich, die für die vorliegende Problemstellung eine nennenswerte Rolle spielen:

- Förderung der Verwendung eines definierten, systemweit konsistenten Vokabulars

$\rightarrow$ Positiver Einfluss auf die Kommunikation zwischen verschiedenen Organisationen

- Visualisierung von Systemspezifikation und -entwurf in Diagrammen

- Berücksichtigung mehrerer interagierender Aspekte und Sichten auf ein System

- Unterstützung der Analysetätigkeit von Systemen durch eine definierte Disziplin

- Validierung von Systemdesign-Aspekten durch Animation

- Schrittweise Verfeinerung des Systemmodells von einer Black-Box Sicht hin zu einer White-Box Sicht, mit einer entsprechenden Erstellung von Testfällen

Bildet nun das Modell im Systems Engineering die Grundlage für alle Systemartefakte, dann spricht man vom modellbasierten Systems Engineering, kurz MBSE [4]. Beim MBSE handelt es sich um eine formalisierte Anwendung der Modellierung zur Unterstützung von Systemanforderungen, Entwurf, Analyse, Verifikation und Validierung über den gesamten Produktlebenszyklus [8]. Abbildung 1 beschreibt nach [11] die drei Säulen Methode, Sprache und Tool, die der Anwendung von MBSE zugrunde liegen. Damit wird auch die eingangs beschriebene Tatsache, dass eine erfolgreiche Entwicklung eine formale Sprache und Methode benötigt im Rahmen einer Toolanwendung erfüllt.

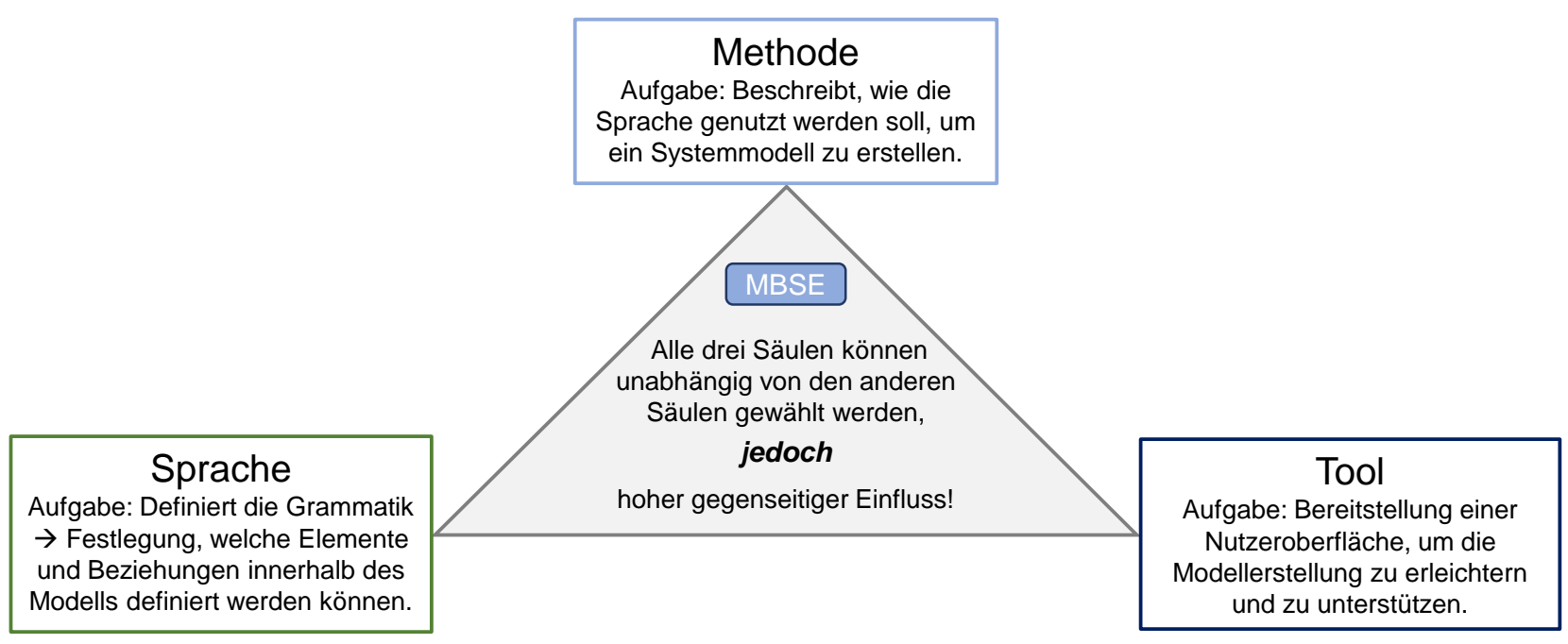

Bild 1: Die drei Säulen des MBSE nach [11] 


\subsection{Anforderungsmanagement}

Das Anforderungsmanagement lässt sich grundsätzlich als Prozess zur Erfassung, Rückverfolgung und Verwaltung von Stakeholder-Bedürfnissen beschreiben, wobei Änderungen und Ableitungen über den gesamten Lebenszyklus hinweg berücksichtigt werden [9]. Ein Stakeholder ist dabei als Person, Organisation oder Sache zu verstehen, die ein Interesse an dem zu entwickelnden System oder Projekt hat oder davon beeinflusst wird [4]. In [9] und [12] wird der Begriff Requirements Engineering als Ansatz für das Anforderungsmanagement und die Spezifikation verwendet. Die darin beschriebenen Definitionen und Zielsetzungen dienen in dieser Arbeit unter anderem als Grundlage für die Kriteriendefinition und sind in der folgenden Abbildung 2 dargestellt. Die Definition unterschiedlicher Abstraktionsebenen in der Abbildung bedeutet dabei, dass die Anforderungen in Ebenen strukturiert werden können, die sich im Abstraktionsgrad unterscheiden und gleichzeitig die Zufriedenheitsbeziehung zwischen diesen Ebenen rückverfolgbar ist. Dadurch entsteht ein Verifizierungszusammenhang zwischen den einzelnen Ebenen, womit ein präzises Mittel zur Qualifizierung von Lösungen zur Verfügung steht [9].

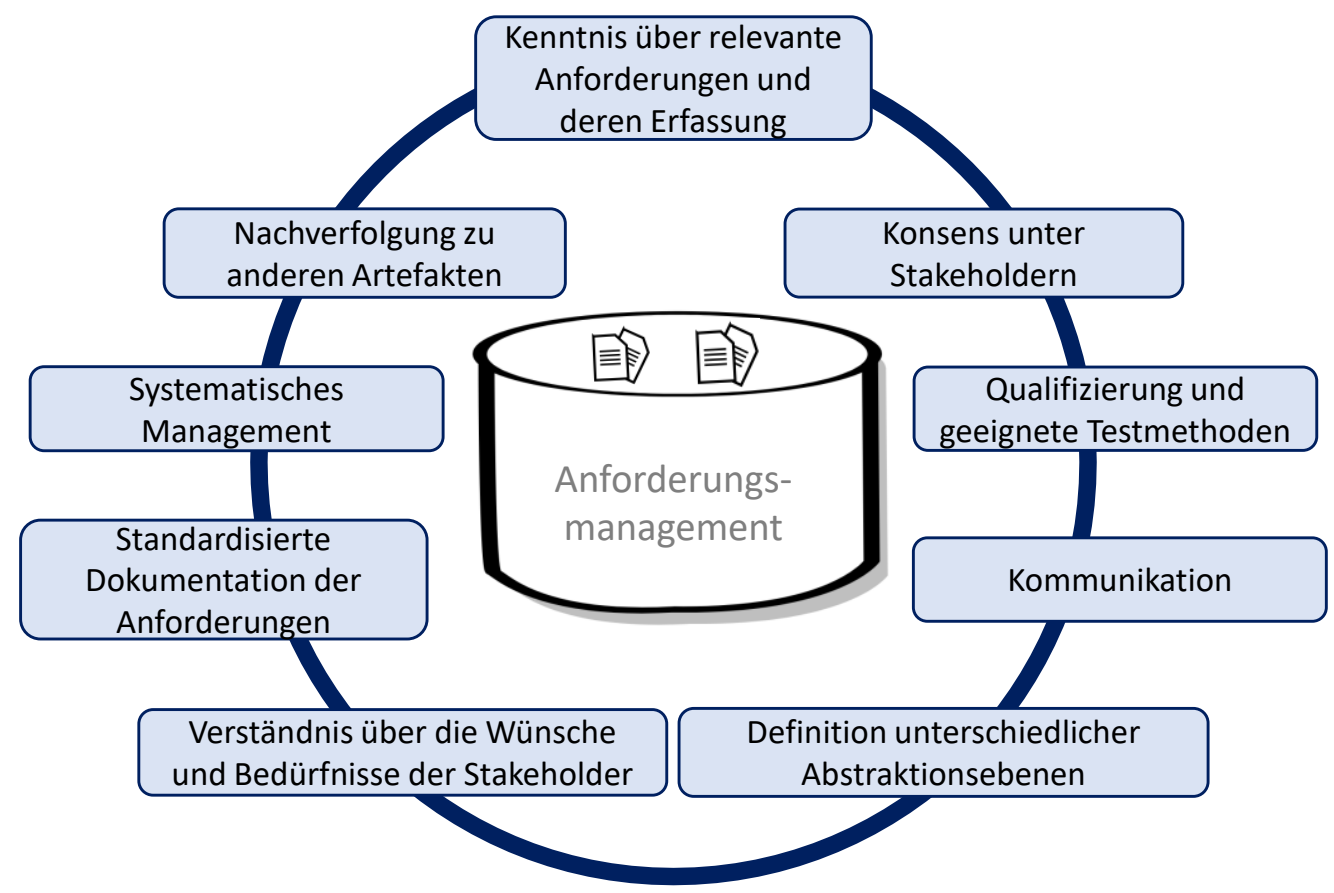

Bild 2: Definition und Zielsetzung Anforderungsmanagement nach [9] und [11]

\subsection{Experimentalsatellit}

Eine Motivation für die Entwicklung eines Experimentalsatelliten ist die Gewinnung von Erkenntnissen bezüglich des Einsatzes neuartiger Kommunikationstechnologien und deren Verhalten in einer bestimmten Betriebsumgebung. Dabei spielt die Identifikation von Möglichkeiten und Einschränkungen für den entsprechenden Nutzlasteinsatz, aber auch für den Satelliten selbst eine wichtige Rolle. Abbildung 3 zeigt im linken Feld einen dafür einsetzbaren Kommunikationsnutzlast-Umfang. Es gilt dabei die Bedürfnisse der Kommunikationsnutzer zu erfüllen, was die Koordination und Partizipation vieler technischer Bereiche mit sich bringt [13]. Des Weiteren bietet ein Experimentalsatellit die Möglichkeit, zusätzliche Erkenntnisse bezüglich der Einsatzmöglichkeiten neuartiger Satellitentechnik in einem speziellen Betriebsumfeld zu gewinnen. Die Bedürfnisse dieser Nutzer sind bei der Entwicklung des Satelliten ebenfalls entsprechend zu berücksichtigen. Abbildung 3 zeigt im rechten Feld einen möglichen Umfang an Experimenten für entsprechende Untersuchungen. 


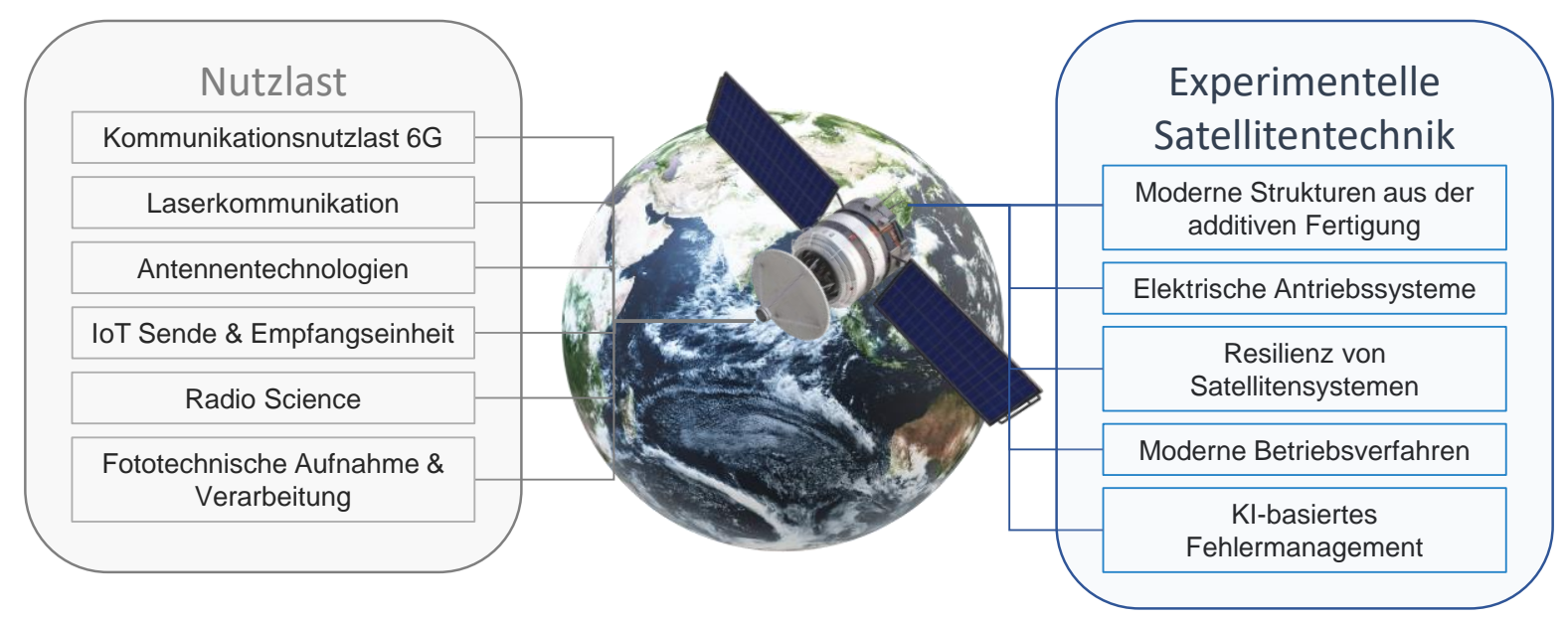

Bild 3: Experimenteller Umfang eines Experimentalsatelliten

\section{Forschungsvorgehen}

Das Vorgehen zur Auflösung der Problemstellung und zur Erfüllung des Forschungsziels ist in drei Arbeitsabschnitte zu gliedern. Im ersten Abschnitt wurden auf Grundlage von Interviews, Diskussionsrunden und einer Literaturrecherche aussagekräftige und konsistente Kriterien ermittelt, die als Grundlage für die Beurteilung der eruirten Ansätze dienen. Im zweiten Arbeitsabschnitt wurde eine systematische Literaturrecherche durchgeführt. Die methodische Grundlage dafür bildet die in sechs Schritte gegliederte Vorgehensmethodik nach [14]. Die in Tabelle 1 dargestellte Suchmatrix zeigt, welche Begriffe der Suchlogik zugrundeliegen. Diese Matrix wurde in einen Suchstring übersetzt und in die Scopus Datenbank eingefügt. Als Ergebnis wurden 867 Artikel identifiziert, die dann wiederum in einem mehrschrittigen Auswahlverfahren auf inhaltliche Relevanz geprüft wurden. Als Ergebnis wurden 26 Artikel identifiziert, die eine Herangehensweise für die modellbasierte Umsetzung des Anforderungsmanagements erörtern. Anschließend wurde eine detaillierte Analyse der Inhalte der Artikel durchgeführt, mit dem Resultat, dass 14 Arbeiten eine Abhandlung beinhalten, die entweder für die vorliegende Forschungsfrage eine Relevanz haben oder im Hinblick auf die Problemstellung für weiterführende Untersuchungungen im Rahmen von wissenschaftlichen Arbeiten erwähnsnswert sind. Abschließend wurden im dritten Arbeitsschritt die eruierten Ansätze und Inhalte anhand der im ersten Arbeitsschritt ausgearbeiteten Kriterien beurteilt, sowie deren Möglichkeiten und Einschränkungen abgeleitet.

\begin{tabular}{|c|c|c|c|}
\hline \multicolumn{2}{|c|}{} & \multicolumn{3}{c|}{ Aspekt } \\
\cline { 2 - 4 } & Anforderung $^{*}$ & Management & MBSE \\
\cline { 2 - 4 } & Requirement $^{*}$ & Engineering & Model based systems engineering \\
\cline { 2 - 4 } & & & Modellbasiertes Systems Engineering \\
\cline { 2 - 4 } & & & SysML \\
\hline
\end{tabular}

Tabelle 1: Suchmatrix für die Literaturrecherche

\section{Modellbasierte Ansätze für das Anforderungsmanagement}

\subsection{Herleitung Bewertungskriterien}

Im Folgenden werden die Beurteilungskriterien definiert, auf deren Grundlage die Bewertung der Ansätze durchgeführt wird. Die Beurteilungskriterien sind in vier Kategorien eingeteilt. Die erste Kategorie umfasst Kriterien, die auf der Zielsetzung des Systems-Engineering beruhen und aus [8] abgeleitet wurden. Die zweite Kategorie beinhaltet Kriterien, die wesentliche Aspekte des Anforderungsmanagements enthalten und auf Grundlage der Ansätze und Aussagen von [4], [9] und [12] definiert wurden. Die dritte Kategorie fasst Randbedingungen 
zusammen, die sich aus dem Satellitenprojekt ergeben. Diese Randbedingungen wurden sowohl durch Interviews mit den domänenspezifischen Entwicklern, als auch dem Entwicklungsteam der übergreifenden Experimentalsatelliten-Plattform identifiziert und zu den entsprechenden Kernaussagen zusammengefasst. In der vierten Kategorie sind allgemeine Aspekte enthalten, die den drei vorher genannten Kategorien nicht zuzuordnen sind, jedoch eine Relevanz für die Umsetzung eines modellbasierten Anforderungsmanagements haben. Abbildung 4 zeigt die Beurteilungskriterien.

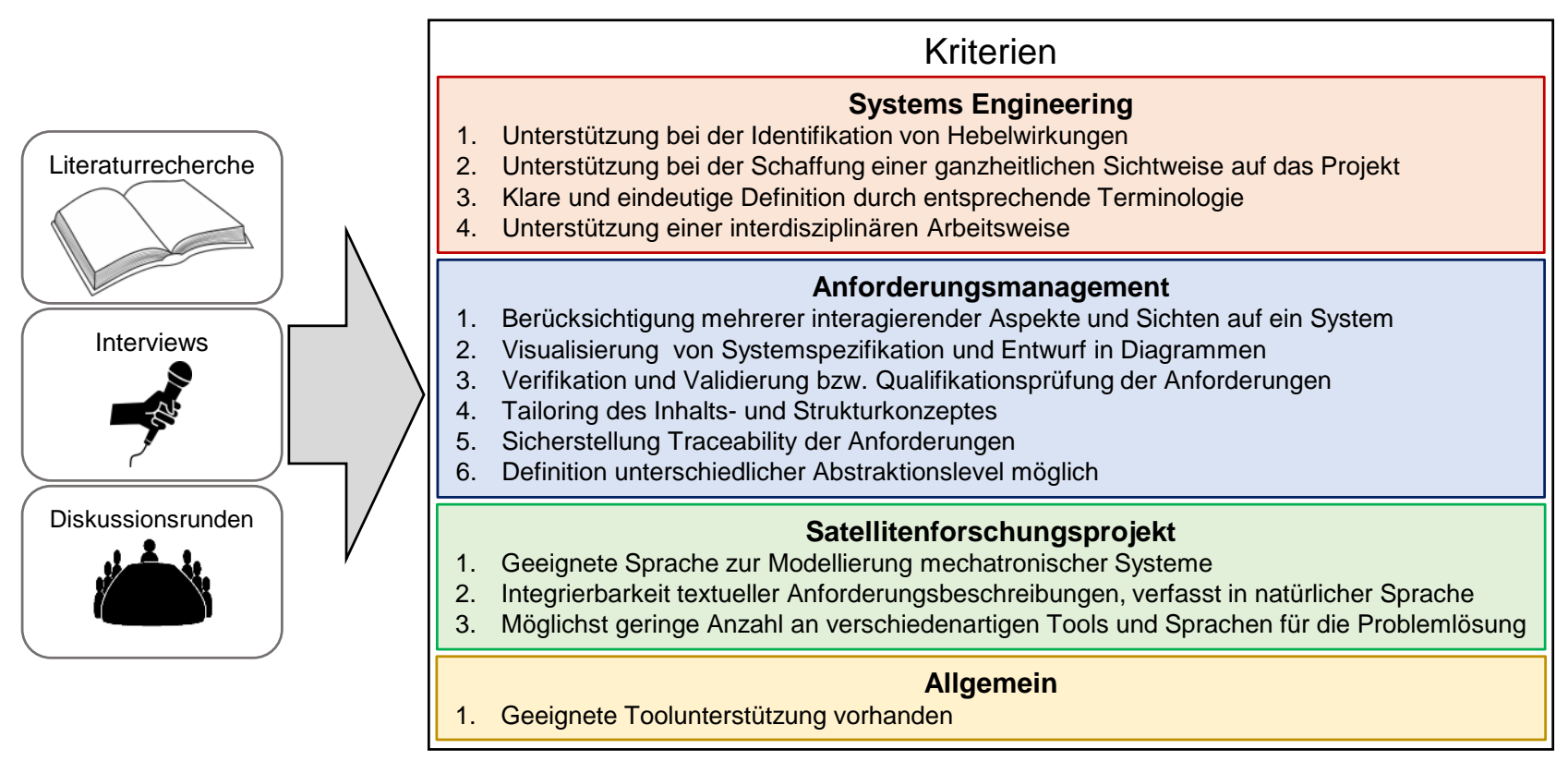

Bild 4: Beurteilungskriterien für die Bewertung der identifizierten Ansätze

\subsection{Ausgeklammerte Ansätze und Abhandlungen}

Die Inhalte der folgenden Arbeiten wurden für die Ableitung eines modellbasierten Ansatzes für das Anforderungsmanagement nicht berücksichtigt, sind jedoch im Rahmen der durchgeführten Literaturrecherche erwähnenswert, da sie im Allgemeinen Aspekte zur Auflösung der beschriebenen Problemstellung untersuchen. Bis auf [15] erörtern die betrachteten Ansätze entweder einen modellbasierten Ansatz oder es liegt ein modellbasierter Ansatz für die Problemlösung und Beantwortung der Forschungsfrage zugrunde. In [15] wird dagegen die Vorgehensweise für eine investigative Recherche zur Auflösung der Fragestellung, wie sich die Einführung von MBSE auf den Anforderungs-Engineering-Prozess im Rahmen eines Akquisitionsprozesses auswirkt, erörtert und ist daher im Rahmen der hier durchgeführten Literaturrecherche erwähnenswert. Die Arbeit von [5] beschreibt einen Ansatz für das Anforderungsmanagement und -engineering für ein Systems of Systems. Ein System of Systems (SoS) wird dabei als ein Metasystem-Gebilde verstanden, in welchem eine operative und betriebswirtschaftliche Unabhängigkeit von Elementen vorliegt. Die Elemente des SoS sind in der Lage unabhängig voneinander zu operieren [5]. Durch diese betriebliche und verwaltungstechnische Unabhängigkeit der Komponentensysteme ist es notwendig, die Anforderungen sowohl auf Systemebene, als auch auf der SoS Metaebene zu betrachten und zu verwalten, womit Inkonsistenzen ausgeschlossen werden sollen [5]. Die Grundlage zur Auflösung der Problemstellung bildet dabei der kontextbasierte Anforderungs-Engineering Ansatz, genannt ACRE (approach for context-based requirements engineering). Da es sich bei der in dieser Arbeit vorliegenden Problemstellung und Forschungsfrage um eine Betrachtung auf Systemebene handelt und nicht um ein System of Systems, wird der in [5] beschriebene Ansatz nicht weiter betrachtet, beinhaltet jedoch interessante Erkenntnisse für 
die Umsetzung eines modellbasierten Ansatzes für das Anforderungsmanagement auf einer höheren Metaebene. Des Weiteren sind die Arbeiten [16], [17] und [18] ebenfalls auszuklammern, da sie die modellbasierte Umsetzung eines zieleorientierten Anforderungsmanagements erörtern. Das zieleorientiere Anforderungsmanagement GORE (goal-oriented Requirements engineering) ermöglicht die Modellierung soziotechnischer Aspekte und nicht funktionaler Anforderungen. Die Nachvollziehbarkeit zwischen Anforderungen und Stakeholder-Zielen, die Tradeoff-Analyse zwischen widersprüchlichen Zielen, die Unterstützung von adaptivem Verhalten, die Validierung mit Management-Stakeholdern und die ganzheitliche Entscheidungsfindung stehen beim GORE im Fokus [17]. Für die Umsetzung von GORE steht die zielorientierte Sprache KAOS zur Verfügung, die eine Erweiterung des SysML Anforderungsmodells darstellt und einen leistungsfähigen Satz von Konzepten und Spezifikationen von Zielmodellen bietet [16]. Eine kurze Erläuterung der Modellierungssprache SysML folgt im nächsten Kapitel. Die drei Arbeiten sind dahingehend im Kontext dieser Arbeit erwähnenswert, dass die Umsetzung eines zieleorientierten Anforderungsmanagements im Rahmen einer Modellerweiterung zu einem späteren Zeitpunkt der Modellierungsphase des Experimentalsatelliten-Projektes durchaus sinnvoll erscheint.

\subsection{Ergebnisdarstellung der Bewertung}

Die Analyse und Bewertung der verbliebenen Ansätze auf Grundlage der Bewertungskriterien ergab, dass bei allen Arbeiten die Grundätze des Systems-Engineering herangezogen und berücksichtigt werden. Die Schaffung einer ganzheitlichen Sichtweise auf die jeweilige Problemstellung und eine klare und eindeutige Terminologie spielen bei den betrachteten Arbeiten eine wichtige Rolle. Die Arbeiten [1], [19], [20], [21] und [22] beschreiben die grundlegenden Möglichkeiten eines Konzeptes für die Umsetzung eines modellbasierten Anforderungsmanagements für einen Experimentalsatelliten. Aus den Inhalten dieser Arbeiten lässt sich ein umsetzungsfähiger Ansatz für das modellbasierte Anforderungsmanagement ableiten, der zugleich alle Bewertungskriterien erfüllt. Abbildung 5 zeigt eine Bewertungsmatrix für die fünf Ansätze auf Grundlage der in Kapitel 4.1 definierten Kriterien. Die in Abbildung 5 dokumentierte Systems Modeling Language (SysML) ist ein Profil der Unified Modeling Language (UML), das von der Object Management Group (OMG) in Zusammenarbeit mit dem International Council on Systems Engineering (INCOSE) standardisiert wurde. Während sich die UML hauptsächlich auf die Modellierung und Analyse von Software konzentriert, adressiert die SysML Systeme, die Hardware und Software mischen können, wie z. B. cyberphysikalische Systeme (CPS) oder aber auch die vorher erläuterten Systems of systems (SoS). SysML ist eine erweiterte Teilmenge der UML 2, die Sequenz-, Zustandsmaschinen-, Anwendungsfall- und Paketdiagramme wiederverwendet und zusätzlich Aktivitätsdiagramme, Klassendiagramme und zusammengesetzte Strukturdiagramme modifiziert und zwei neue Diagrammtypen hinzufügt, nämlich parametrische Diagramme und Anforderungsdiagramme [17]. Resultierend aus dem in der Abbildung 5 gezeigtem Ergebnis der Literaturrecherche lässt sich SysML als Sprache für die Umsetzung des modellbasierten Anforderungsmanagements ableiten. Sie bietet die erforderlichen Möglichkeiten, um die in der Einleitung geschilderte Problemstellung beherrschbar zu machen und zur Auflösung beizutragen. Weitere Ansätze in denen SysML für die Modellierung von Systemanforderungen herangezogen wird, sind in [23] und [24] geschildert. Letzere Arbeit implementiert einen modellbasierten Ansatz unter Nutzung von SysML und den Grundlagen des Systems-Engineering für die Entwicklung eines Prozesses zur Anforderungsanalyse für das Designproblem in der Flugzeugentwicklung. Zur Ableitung einer geeigneten Methode und zur Identifikation eines geeigneten Tools werden neben den Ergebnissen aus [13] und [25] und den in der Abbildung 5 aufgelisteten Arbeiten die Ergebnisse der Arbeiten [26] und [27] herangezogen. Die in [27] geschilderte Methode MBSE-Grid ist durch die Analyse verschiedener MBSE Methoden (z.B. Harmony, OOSEM, Vitech), Frameworks und deren Anwendung auf reale Projekte entstanden. Im Vergleich zu 
diesen Methoden gibt die Methode MBSE-Grid eine eindeutige Richtlinie vor und schlägt einen vereinfachten Ansatz für die Modellierung mittels der Sprache SysML vor [27]. Eine vereinfachte Modellierung ist ein nennenswerter Aspekt bei der Unterstützung einer interdisziplinären Arbeitsweise - was ein wichtiges Bewertungskriterium darstellt (siehe Bild 4) - da es Hürden bei der Nutzungsakzeptanz abbaut und leichter in den Entwicklungsprozess implementiert werden kann. Die Umsetzung der MBSE-Grid Methode unter Nutzung der SysML Sprache wird in [26] im Rahmen einer systematischen Evaluierung von Anforderungsmanagement-Tools im MagicDraw Tool verwendet. Dabei werden Vorteile wie eine visuelle Darstellung mittels benutzerdefinierter Diagramme und einem großen Portfolio an Beziehungsdefinitionen hervorgehoben, was unter anderem das Tailoring des Inhalts- und Strukturkonzeptes fördert und das Kriterium der Visualisierung der Systemspezifikation in Diagrammen erfüllt (vgl. Bild 4).

\begin{tabular}{|c|c|c|c|c|c|c|}
\hline & & [1] & [19] & [20] & [21] & [22] \\
\hline Kategorie & Kriterium & & & & & \\
\hline \multirow{4}{*}{$\begin{array}{l}\text { Systems- } \\
\text { Engineering }\end{array}$} & SE1 & 0 & 0 & 0 & 0 & 0 \\
\hline & SE2 & 0 & 0 & 0 & 0 & 0 \\
\hline & SE3 & 0 & 0 & 0 & 0 & 0 \\
\hline & SE4 & 0 & 0 & 0 & 0 & 0 \\
\hline \multirow{6}{*}{$\begin{array}{l}\text { Anforderungs- } \\
\text { management }\end{array}$} & AM1 & 0 & 0 & 0 & 0 & 0 \\
\hline & AM2 & 0 & 0 & 0 & 0 & 0 \\
\hline & AM3 & 0 & 0 & 0 & 0 & 0 \\
\hline & AM4 & 0 & 0 & 0 & 0 & 0 \\
\hline & AM5 & 0 & 0 & 0 & 0 & 0 \\
\hline & AM6 & 0 & 0 & 0 & 0 & 0 \\
\hline \multirow{3}{*}{ Satellitenprojekt } & SAT1 & SysML & SysML & SysML & SysML & SysML \\
\hline & SAT2 & 0 & 0 & 0 & 0 & 0 \\
\hline & SAT3 & 0 & 0 & 0 & 0 & 0 \\
\hline Allgemein & $\mathrm{AL} 1$ & $\otimes$ & Papyrus & Papyrus & Harmony SE & $\otimes$ \\
\hline
\end{tabular}

Bild 5: Bewertungsmatrix für die fünf betrachteten Ansätze

\subsection{Ableitung eines Ansatzes}

Abbildung 6 zeigt anschaulich den abgeleiteten Ansatz als Ergebnis der Literaturrecherche. Es ist erkennbar, dass die SysML als Modellierungssprache herangezogen wird, um die Beziehungen innerhalb des Modells zu definieren. Die Logik des Modellaufbaus beruht auf der MBSE-Grid Methode, womit diese Methode beschreibt, in welcher Art die Anforderungen im Modell implementiert und mit anderen Objekten und Strukturen verknüpft werden können. Schlussendlich erfolgt die Umsetzung der Modellierung mittels des Tools MagicDraw. Die Ersterfassung der Anforderungen und Stakeholder-Bedürfnisse erfolgt mittels eines eigens dafür programmierten Excel-Tools. Die aufgenommenen Informationen sind standardisiert und erfüllen die in [12] und [28] definierten Anforderungen an eine Anforderung. Grund für die Verwendung eines Excel-Tools mit integrierter GUI (graphical user interface) ist die 
Erleichterung der Erfassungstätigkeit in der Anfangsphase und die Reduzierung von möglichen Fehlerquellen im Modellierungsprozess durch unsachgemäße und unsaubere Handhabung und Modellierungstätigkeit.

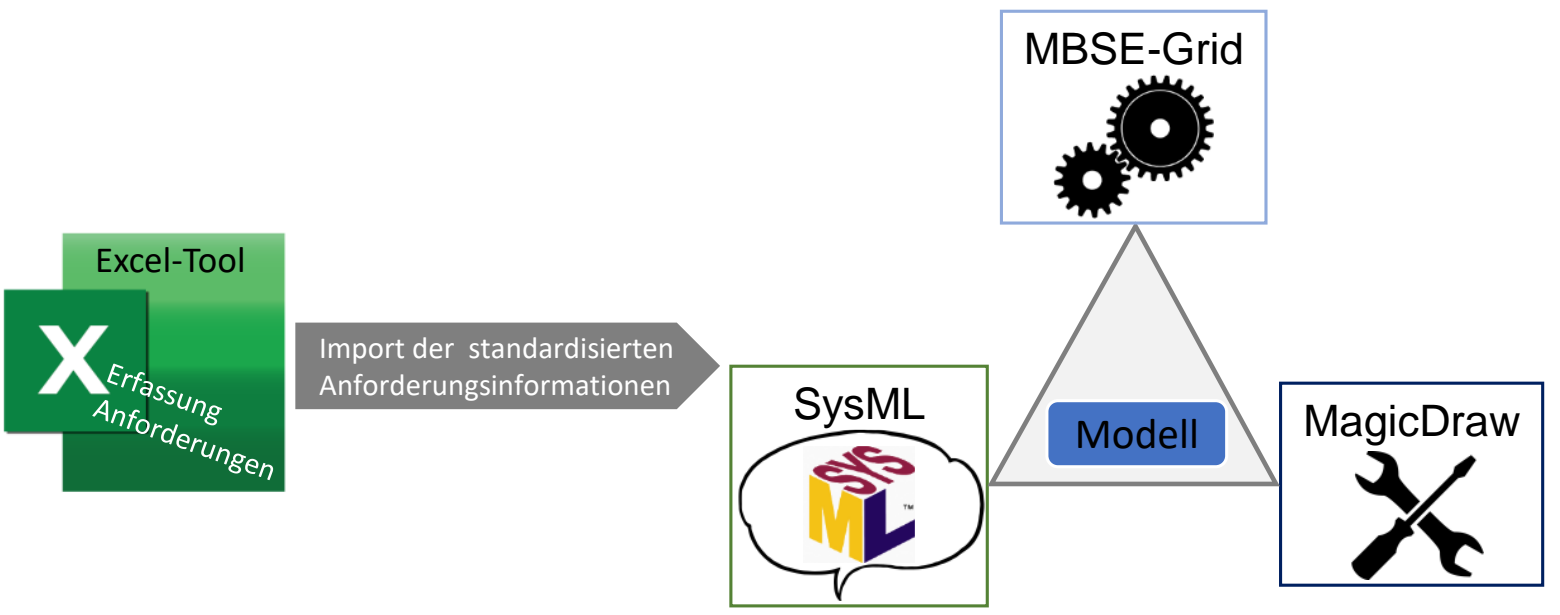

Bild 6: Schematische Darstellung des abgeleiteten Ansatzes

\section{Zusammenfassung und Ausblick}

In dieser Arbeit wurde auf Grundlage von elaborierten Bewertungskriterien die Frage erörtert, welche Möglichkeiten und Einschränkungen bisherige Ansätze aus der Literatur zur Umsetzung eines modellbasierten Anforderungsmanagements in einem Satellitenprojekt bieten, um auf dieser Grundlage einen Ansatz für die Umsetzung eines entsprechenden modellbasierten Anforderungsmanagements für ein Satellitenforschungsprojekt abzuleiten. Die Basis für die Bewertungskriterien bilden die Grundsätze des Systems-Engineering, die Zielsetzung des Anforderungsmanagements und die Randbedingungen aus dem Satellitenforschungsprojekt. Als Ergebnis aus der Literaturrecherche leitet sich ein Ansatz ab, dem die SysML als Sprache, die MBSE-Grid als Methode und MagicDraw als Tool zugrunde liegen. Die Anforderungen werden vorab mittels eines Excel-Tools erfasst und dann anschließend in die Modellumgebung importiert. Der nächste Schritt wird die Umsetzung dieses Ansatzes im Rahmen des Satellitenforschungsprojektes sein. Darauffolgend wird eine Validierung des vorgestellten Ansatzes durchgeführt.

\section{Danksagung}

Die vorliegende Forschungsarbeit wurde im Rahmen des Projekts SeRANIS - Seamless Radio Access Networks in the Internet of Space durchgeführt. Das Projekt wird gefördert durch das dtec.bw - Forschungszentrum Digitalisierung und Technik der Bundeswehr, mit dem Förderkennzeichen 150009910.

\section{Literaturverzeichnis}

[1] F. Hayat, M. W. Anwar, F. Azam, and A. Kiran, "A SYSML-Based Approach for Requirements Risk Management and Change Control," in Proceedings of the 201911 th International Conference on Information Management and Engineering, London United Kingdom, 09192019, pp. 20-24.

[2] J. Kößler and K. Paetzold, "Integration of MBSE into existing development processes - Expectations and challenges," in Proceedings of the 21st International Conference on Engineering Design (ICED 17) Vol 3: Product, Services and Systems Design, Vancouver, Canada.

[3] Y. Bernard, "Requirements management within a full model-based engineering approach," Syst. Engin., vol. 15, no. 2, pp. 119-139, 2012, doi: 10.1002/sys.20198.

[4] J. Holt, S. A. Perry, and M. Brownsword, Model-based requirements engineering. Stevenage Herts U.K.: Institution of Engineering and Technology, 2012. 
[5] J. Holt, S. Perry, R. Payne, J. Bryans, S. Hallerstede, and F. O. Hansen, "A Model-Based Approach for Requirements Engineering for Systems of Systems," IEEE Systems Journal, vol. 9, no. 1, pp. 252-262, 2015, doi: 10.1109/JSYST.2014.2312051.

[6] F. Jakob, S. Mazzini, and A. Jung, "A SysML-Based Methodology in a Concurrent Satellite Design Process," in SAE Technical Paper Series, 2011.

[7] O. A. Yarom, J. Zhang, C. Raulf, X. Liu-Henke, and T. Vietor, "Anforderungsmanagement für die modellbasierte Entwicklung mechatronischer Systeme im digitalisierten und vernetzten Umfeld," in Proceedings ASIM SST 2020, Oct. 2020, pp. 213-220.

[8] David D. Walden, Garry J. Roedler, Kevin J. Forsberg, and R. Douglas Hamelin and Thomas M. Shortell, "INCOSE Systems Engineering Handbook: A Guide for System Life Cycle Processes and Activities,"

[9] J. Dick, E. Hull, and K. Jackson, Requirements Engineering. Cham: Springer International Publishing, 2017.

[10] U. Lindemann, Methodische Entwicklung technischer Produkte. Berlin, Heidelberg: Springer Berlin Heidelberg, 2009.

[11] L. Delligatti, SysML Distilled: A Brief Guide to the Systems Modeling Language. United States: U.S. Corporate and government Sales, 2013.

[12] C. Rupp, Requirements-Engineering und -Management: Aus der Praxis von klassisch bis agil, 6th ed. München: Hanser, 2014.

[13] S. Gao, W. Cao, L. Fan, and J. Liu, "MBSE for Satellite Communication System Architecting," IEEE Access, vol. 7, pp. 164051-164067, 2019, doi: 10.1109/ACCESS.2019.2952889.

[14] L. A. Machi and B. T. M. McEvoy, The Literature Review: Six steps to success (accessed: May 132021 ).

[15] R. Giachetti, K. Holness, and M. McGuire, "The Ability of Engineers to Extract Requirements from Models," in The Ability of Engineers to Extract Requirementsfrom Models, Banff, AB, 82018, pp. 394-399.

[16] M. Ahmad, J.-M. Bruel, R. Laleau, and C. Gnaho, "Using RELAX, SysML and KAOS for Ambient Systems Requirements Modeling," Procedia Computer Science, vol. 10, pp. 474-481, 2012, doi: 10.1016/j.procs.2012.06.061.

[17] D. Amyot, A. A. Anda, M. Baslyman, L. Lessard, and J.-M. Bruel, "Towards Improved Requirements Engineering with SysML and the User Requirements Notation," in 2016 IEEE 24th International Requirements Engineering Conference (RE), Beijing, China, 92016, pp. 329-334.

[18] R. Laleau, F. Semmak, A. Matoussi, D. Petit, A. Hammad, and B. Tatibouet, "A first attempt to combine SysML requirements diagrams and B," Innovations Syst Softw Eng, vol. 6, 1-2, pp. 47-54, 2010, doi: 10.1007/s11334-009-0119-y.

[19] M. Borsato, N. Wognum, M. Peruzzini, J. Stjepandić, and W. J. C. Verhagen, Eds., Driving Product Design and Requirements Management with SysML. Amsterdam: IOS Press, 2016.

[20] C.-H. Chang, C.-W. Lu, W. C.-C. Chu, P.-A. Hsiung, and D.-M. Chang, "SysML-Based Requirement Management to Improve Software Development," Int. J. Soft. Eng. Knowl. Eng., vol. 26, no. 03, pp. 491511, 2016, doi: 10.1142/S0218194016500200.

[21] Z. Chen, Y. Wang, Y. Zhang, and T. Li, "MBSE Approach to Aero-Engine Turbine System Design and Requirements Management," in Lecture Notes in Electrical Engineering, The Proceedings of the 2018 AsiaPacific International Symposium on Aerospace Technology (APISAT 2018), X. Zhang, Ed., Singapore: Springer Singapore, 2019, pp. 1730-1744.

[22] A. H. F. Laender, Conceptual modeling -- ER 2009: 28th International Conference on Conceptual Modeling, Gramado, Brazil, November 9-12, 2009 proceedings. Berlin, New York: Springer, 2009.

[23] Michel dos Santos Soares, Jos Vrancken, Proceedings of 2007 IEEE International Conference on Systems, Man and Cybernetics: The Delta Centre-Ville at Montréal, Quebec, Canada October 7-10, 2007/ IEEE. Montreal, Quebec: Omni Press, 2007.

[24] E. Karagoz, K. A. Reilley, and D. N. Mavris, "Model-Based Approach to the Requirements Analysis for a Conceptual Aircraft Sizing and Synthesis Problem," in AIAA Scitech 2019 Forum, San Diego, California, 01072019.

[25] M. Omiciuolo, K. Paetzold, and R. Förstner, General Purpose Modeling and Domain Specific Simulation: $A$ Framework for Space Mechanisms Design. Piscataway, NJ: IEEE, 2015.

[26] J. M. McLellan, B. Morkos, G. G. Mocko, and J. D. Summers, "Requirement Modeling Systems for Mechanical Design: A Systematic Method for Evaluating Requirement Management Tools and Languages," in Volume 3: 30th Computers and Information in Engineering Conference, Parts $A$ and B, Montreal, Quebec, Canada, 08152010, pp. 1247-1257.

[27] D. Mazeika, A. Morkevicius, and A. Aleksandraviciene, "MBSE driven approach for defining problem domain," in 2016 11th System of Systems Engineering Conference (SoSE), Kongsberg, Norway, 62016, pp. $1-6$.

[28] W. D. Schindel, "11.2.1 Requirements Statements Are Transfer Functions: An Insight from Model-Based Systems Engineering," INCOSE International Symposium, vol. 15, no. 1, pp. 1604-1618, 2005, doi: 10.1002/j.2334-5837.2005.tb00775.x. 